\title{
In situ cryo-electron microscopy of two-dimensional van der Waals magnets
}

Myung-Geun $\mathrm{Han}^{1}$, Yimei Zhu ${ }^{2}$ and Sang-Wook Cheong ${ }^{3}$

${ }^{1}$ Brookhaven National Laboratory, Upton, New York, United States, ${ }^{2}$ Department of Condensed Matter Physics and Materials Science, Brookhaven National Laboratory, Upton, NY, USA, United States, ${ }^{3}$ Rutgers Center for Emergent Materials, Rutgers University, United States

Magnetism in two-dimensional (2D) materials and their heterostructures have been extensively studied due to the fundamental spin physics and applications for spintronics and quantum information sciences [1-3]. Recently, topologically nontrivial spin structures have been reported in various 2D van der Waals magnets, such as $\mathrm{Cr}_{2} \mathrm{Ge}_{2} \mathrm{Te}_{6}, \mathrm{Fe}_{3} \mathrm{GeTe}_{2}, \mathrm{Fe}_{3} \mathrm{Sn}_{2}$, and intercalated $2 \mathrm{H}-\mathrm{NbS}_{2}$ [4-7]. Phase imaging in transmission electron microscopy provides a unique opportunity to study such magnetism at the nanoscale and its dynamics under external stimuli. Using off-axis electron holography and Lorentz microscopy combined with liquid helium cooling and electrical biasing, we study topological magnetic spin states in 2D magnets and their dynamic behaviors under external magnetic and/or electric fields.

Figs. 1a-d show the crystal structure of $2 \mathrm{H}-\mathrm{TaS}_{2}$ intercalated with $\mathrm{Cr}$ in $1 / 3$ composition with respect to Ta. The $\mathrm{Cr}$ atoms occupy the octahedral holes made by $\mathrm{S}$ atoms between two neighboring $\mathrm{TaS}_{2}$ layers, consistent with the $\sqrt{3} \times \sqrt{3}$ structure reported for the $\mathrm{Cr}_{1 / 3} \mathrm{NbS}_{2}$ [7]. In HAADF STEM image (Fig. 1c), the intercalated $\mathrm{Cr}$ atoms are directly observed. This $\mathrm{Cr}$ intercalation breaks inversion symmetry and gives rise to chiral spin arrangement along the $c$-axis due to the competition between ferromagnetic exchange interaction (FMI) and antisymmetric exchange interaction (DMI). Lorentz TEM images in Fig. 1e show the alternating bright and dark stripe patterns emerging upon cooling below $T \sim 120 \mathrm{~K}$, similar to the helical spin states in $\mathrm{Cr}_{1 / 3} \mathrm{NbS}_{2}$ [7]. However, the periodicity of helical spin states in $\mathrm{Cr}_{1 / 3} \mathrm{TaS}_{2}$ was about $15.6 \mathrm{~nm}$, much shorter than $48 \mathrm{~nm}$ in $\mathrm{Cr}_{1 / 3} \mathrm{NbS}_{2}$, indicating stronger DMI with respect to the FMI. When an external magnetic field along the imaging direction is applied, the periodicity scales nonlinearly to form chiral soliton states (separation of dark stripes increases).

Interestingly, we observed the helicity (rotation sense) reversal occurs across a structural planar defect, as shown in Figs. 2a and b. The schematic in Fig. 2c shows the spin arrangements across the planar defects. Atomic resolution STEM image in Fig. $2 \mathrm{~d}$ shows that the $2 \mathrm{H}$ phase is partly interrupted by $1 \mathrm{~T}$ phase; note that the Ta coordinates are mixed with trigonal prismatic $(2 \mathrm{H})$ and octahedral (1T) in the structural defects. Interestingly, the partial $1 \mathrm{~T}$ phase is only found every two $\mathrm{TaS}_{2}$ layers, forming a superstructure doubling the lattice constant along the $c$-axis. Considering the presence of partial 1T phase, the DMI pathways are illustrated in Fig. 2f. For the next nearest neighbor interaction between two $\mathrm{Cr}$ atoms in $2 \mathrm{H}-\mathrm{TaS}_{2}$ phase, two $\mathrm{S}$ atoms are off-centered from the 2-fold rotation axis, giving rise to a net DMI along the $c$-axis [8]. However, the $\mathrm{S}$ atoms in $1 \mathrm{~T}$ phase restore inversion symmetry at the center of the $\mathrm{Cr}-\mathrm{Cr}$ path, vanishing the DMI. This local vanishing DMI at the planar defects effectively changes the DMI sign to reverse the helicity of helical spin states and chiral solitons in $\mathrm{Cr}_{1 / 3} \mathrm{TaS}_{2}$.

In our cryo-Lorentz phase microscopy study, we directly observed the helicity reversal behavior of the soliton lattices in $\mathrm{Cr}_{1 / 3} \mathrm{TaS}_{2}$ across structural planar defects. Using atomic resolution imaging, we 
show that a partial $1 \mathrm{~T}$ phase in $2 \mathrm{H}-\mathrm{TaS}_{2}$, forming a superstructure with doubling lattice constant along the $c$-axis, breaking local DMI symmetry, and leading to the spin helicity reversal. Our experimental results show that manipulation of structural phases in intercalated 2D magnets provides a viable way to control the helicity of topologically nontrivial soliton lattices.

This work was supported by the US Department of Energy, Basic Energy Sciences, Materials Sciences and Engineering Division (Contract No. DE-SC0012704). TEM sample preparation was carried out at the Center for Functional Nanomaterials, Brookhaven National Laboratory.
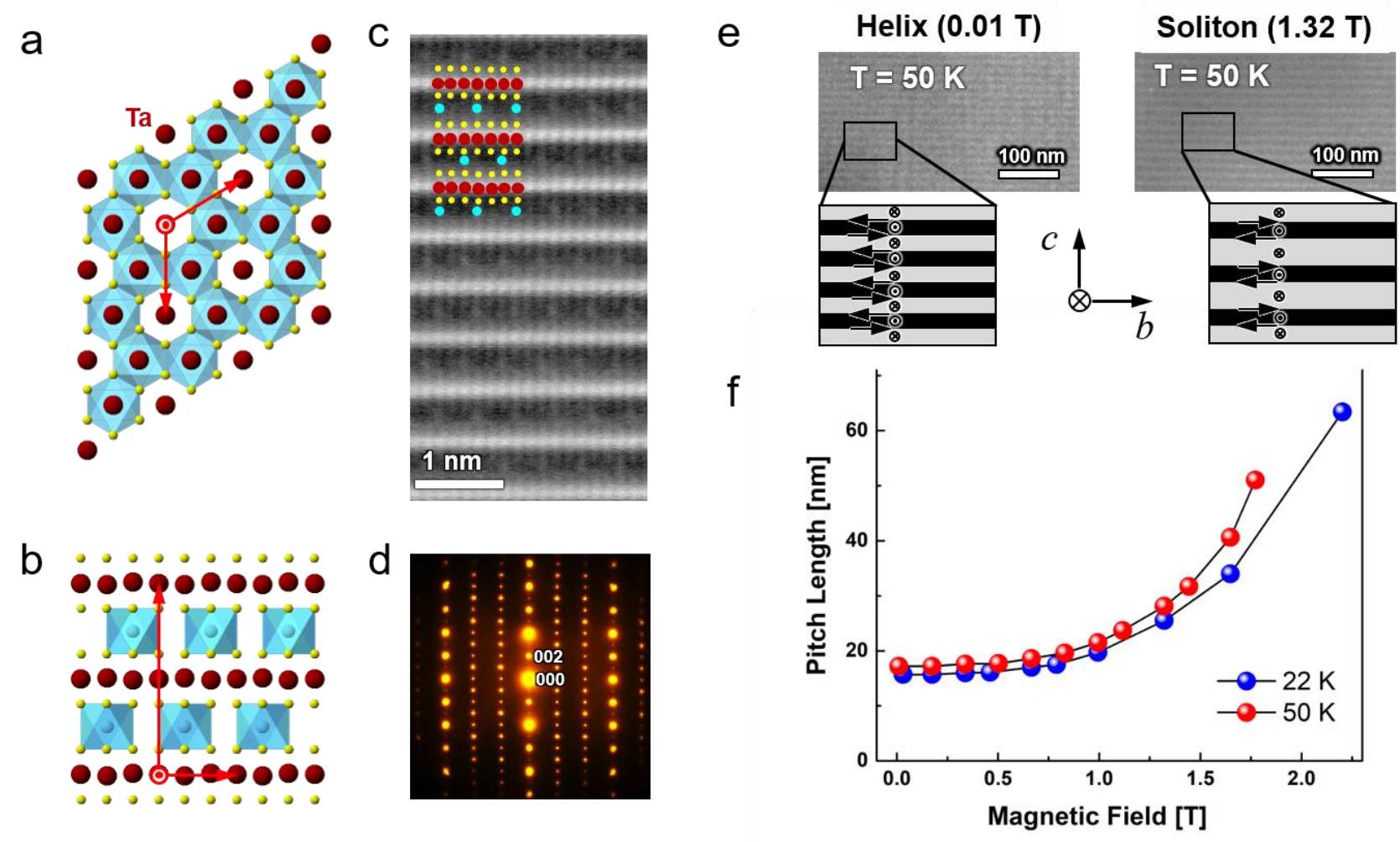

Figure 1. Figure 1| Crystal structures and helical/soliton spin states of $\mathrm{Cr}$-intercalated 2H-TaS2 Atomic models of Cr-intercalated 2H-TaS2 viewed along the c-axis (a), the a-axis (b). HAADF STEM image taken along the a-axis (c) show the $\sqrt{3} \times \sqrt{3}$ Cr site-occupancy. Electron diffraction patterns taken along the a-axis (d) shows the superlattice peaks associated with Cr-intercalation. Lorentz TEM images (e) obtained at $50 \mathrm{~K}$ under various magnetic fields show helical spin states with modulation period $\sim 15.6 \mathrm{~nm}$, that are nonlinearly scaled with increasing magnetic field (f). External magnetic field direction is along the imaging direction (the a-axis). 

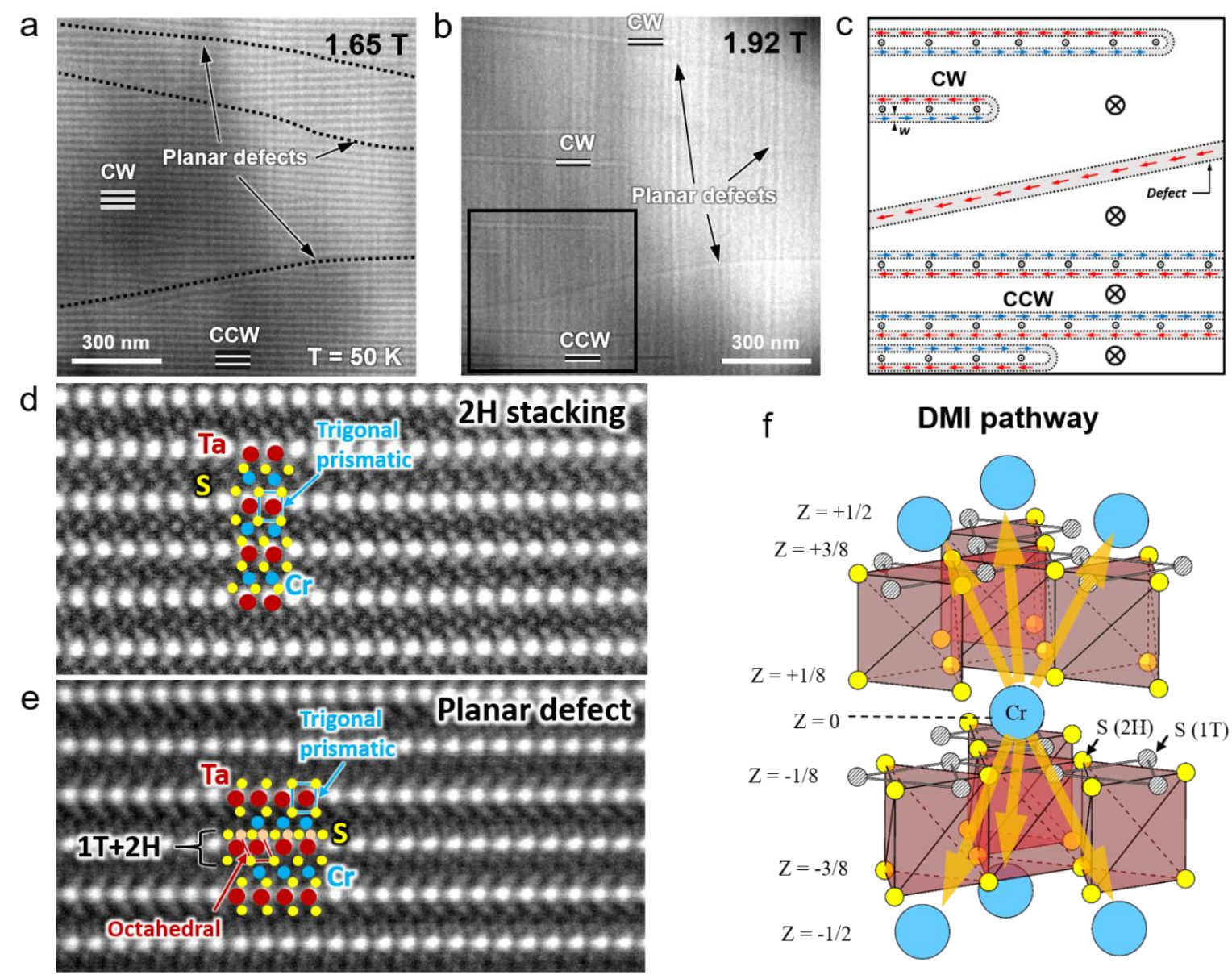

Figure 2. Figure 2| Helicity reversal across planar defects Lorentz TEM images (a-b) under high magnetic field shows reversal of spin helicity across planar defects. Schematic of spin structures (c) in the rectangular box in b. HAADF STEM image taken along the [210] direction shows $2 \mathrm{H}$ stacking (d). At the defect, a partial $1 \mathrm{~T}$ mixing with $2 \mathrm{H}$ stacking as seen by $\mathrm{S}$ atomic column splitting was found. The schematic (f) shows the modified DMI pathway (six arrows), responsible for the spin helicity reversal observed in $\mathrm{b}$. Ta atoms in the center of trigonal prisms are not shown in $\mathrm{f}$. S atoms in the $1 \mathrm{~T}$ phase cancel out interlayer DMI associated arising with $\mathrm{S}$ atoms in $2 \mathrm{H}$ stacking.

References

[1] K. S. Burch, D. Mandrus, and J. Park, Nature 563, 4 (2018).

[2] B. Huang, et al., Nature 546, 270 (2017).

[3] C. Gong, et al., Nature 546, 265 (2017).

[4] M.-G. Han, et al., Nano Lett. 19, 7859-7865 (2019).

[5] T. E. Park, et al., arXiv:1907.01425 (2019).

[6] Q. Du, et al., Advaned Quantum Technologies 2000058 (2020).

[7] Y. Togawa, et al., Physical Review Letters 108, 107202 (2012).

[8] Y. Cao, et al., Materials Today Advances 7, 100080 (2020). 